\title{
Characterization of a ZnSe scintillating bolometer prototype for neutrinoless double beta decay search
}

\author{
M. Tenconi ${ }^{1}$, a, A. Giuliani ${ }^{1,2}$, C. Nones ${ }^{3}$, G. Pessina ${ }^{2}$, O. Plantevin ${ }^{1}$, and C. Rusconi ${ }^{2,4}$ \\ ${ }^{1}$ Centre de Sciences Nucléaires et de Sciences de la Matière, CNRS and Université Paris-Sud, F-91405 Orsay, France \\ ${ }^{2}$ Istituto Nazionale di Fisica Nucleare, Sezione di Milano-Bicocca, I-20126 Milano, Italy \\ ${ }^{3}$ Service de Physique des Particules, CEA Saclay, F-91191 Gif-sur-Yvette, France \\ ${ }^{4}$ Università degli Studi dell'Insubria, Dipartimento di Scienza e Alta Tecnologia, I-22100 Como, Italy
}

\begin{abstract}
As proposed in the LUCIFER project, ZnSe crystals are attractive materials to realize scintillating bolometers aiming at the search for neutrinoless double beta decay of the promising isotope ${ }^{82} \mathrm{Se}$. However, the optimization of the $\mathrm{ZnSe}$-based detectors is rather complex and requires a wide-range investigation of the crystal features: optical properties, crystalline quality, scintillation yields and bolometric behaviour. Samples tested up to now show problems in the reproducibility of crucial aspects of the detector performance. In this work, we present the results obtained with a scintillating bolometer operated aboveground at about $25 \mathrm{mK}$. The detector energy absorber was a single $1 \mathrm{~cm}^{3} \mathrm{ZnSe}$ crystal. The good energy resolution of the heat channel (about $14 \mathrm{keV}$ at $1460 \mathrm{keV}$ ) and the excellent alpha/beta discrimination capability are very encouraging for a successful realization of the LUCIFER program. The bolometric measurements were completed by optical tests on the crystal (optical transmission and luminescence measurements down to $10 \mathrm{~K}$ ) and investigation of the crystalline structure. The work here described provides a set of parameters and procedures useful for a complete pre-characterization of $\mathrm{ZnSe}$ crystals in view of the realization of highly performing scintillating bolometers.
\end{abstract}

\section{Introduction}

Neutrinoless Double Beta decay (0vDBD) is a hypothetical nuclear process violating lepton number by two units, which is possible only in case the neutrino is identical to its own antiparticle. The observation of this phenomenon would thus state the neutrino nature and define the absolute scale and hierarchy of its mass eigenstates, being the decay rate proportional to the square effective neutrino mass. Double beta decay is possible only for a few eveneven isotopes; measured half-lives are in the range of $10^{19}$ to $10^{21}$ years for the two-neutrino mode, while the neutrinoless mode has never been observed and actual limits are of the order of $10^{25}$ years [1]: background reduction is therefore a primary experimental challenge. The sought $0 v \mathrm{DBD}$ footprint is a peak at the transition energy $Q$ of the decay, in the electronic spectrum. While the residual $\beta, \gamma$ background can be abated by operating underground and choosing a candidate isotope with energy transition higher than the environmental radioactivity end-point, degraded $\alpha$ particles from surface contaminations remain dangerous as they spoil the typical energy region of interest for most candidate isotopes: that's why particle discrimination techniques are preferable. Bolometers are optimal detectors to be employed in $0 v \mathrm{DBD}$ experiments [2], thanks to their good energy resolution, efficiency and flexibility in the material choice. Operated at very low temperatures,

\footnotetext{
a e-mail: margherita.tenconi@csnsm.in2p3.fr
}

they can register the temperature signal induced by particle interactions (figure 1). If a scintillating absorber is used, it is possible to exploit double read-out of heat and light to perform event discrimination: $\alpha$ particles emit a different amount of light with respect to $\beta, \gamma$ particles. This technique was chosen for the LUCIFER project [3], aiming at probing $0 v \mathrm{DBD}$ of ${ }^{82} \mathrm{Se}(Q=2996 \mathrm{keV})$ by means of $\mathrm{ZnSe}$ scintillating bolometers.
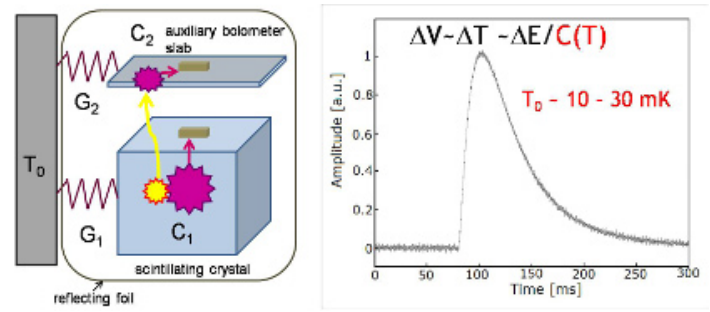

Figure 1. Schematics of a scintillating bolometer. The deposited energy is converted by a thermometer into a voltage pulse. Due to the heat capacity dependence of the signal, typical operating temperatures are $10-30 \mathrm{mK}$. Scintillation light is collected by a thin auxiliary bolometer; a reflecting foil maximizes the collection efficiency, LYs being of some tens of $\mathrm{keV} / \mathrm{MeV}$ or lower.

The $\mathrm{ZnSe}$ is not an intrinsic scintillator; in fact the luminescence is due to crystal imperfections, difficult to reproduce. As a consequence, $\mathrm{ZnSe}$ absorbers are quite complex to characterize: in past tests, important differ- 
ences were observed from sample to sample [4, 5], under several aspects such as bolometric performance, light yield (LY) and the ratio of $\alpha$ 's to $\beta, \gamma$ 's LY (i.e. the quenching factor, QF).

Preliminary characterization is therefore necessary: in this work we provide a series of useful procedures and parameters, having investigated the optical and structure properties of a crystal which exhibited excellent bolometric performances. First, we assembled a prototype scintillating bolometer and operated it aboveground in order to assess energy resolution and $\alpha / \beta, \gamma$ discrimination capability, then we performed optical and X-ray diffraction measurements on the same $\mathrm{ZnSe}$ sample.

\section{Detector setup}

A scintillating bolometer was assembled: the absorber is a $5 \mathrm{~g}$ cubic $1 \times 1 \times 1 \mathrm{~cm}^{3}$ ZnSe sample, provided by Alkor Technologies in Saint Petersbourg (Russia). Two nominally identical light detectors face the crystal on two opposite sides, at approximately $2 \mathrm{~mm}$ distance: they are square hyperpure Ge slabs, $15 \mathrm{~mm}$ side and $0.5 \mathrm{~mm}$ thickness. Thermal signals from all the three absorbers were provided by technically identical $3 \times 1 \times 0.6 \mathrm{~mm}^{3}$ Neutron Transmutation Doped Ge thermistors (NTDs), whose resistivity is described by a law in Variable Range Hopping regime with Coulomb gap,

$$
\rho(T)=\rho_{0} \cdot \exp \left(T_{0} / T\right)^{0.5}
$$

where $\rho_{0}=1.09 \Omega \cdot \mathrm{mm}$ and $T_{0}=3.83 \mathrm{~K}$ were determined in a previous characterization of the same Ge wafer. Mechanical coupling of the crystals to the copper holder is provided by PTFE pieces, while the thermal link is supplied by golden wires, $50 \mu \mathrm{m}$ diameter and about $15 \mathrm{~mm}$ long, which are actually the electrical connections for the thermistors. In order to improve light collection, the interior surface of the copper holder was covered with a reflecting foil.

A smeared uranium source was placed in front of the bottom side of the $\mathrm{ZnSe}$ crystal, so as to provide a continuum spectrum from the nominal energy end-point to lower regions, analogously to the one originated from $\alpha$ surface contaminations. The used source is ${ }^{238} \mathrm{U}$ depleted, emitting alpha particles with $4.47 \times 10^{9}$ y half-life at $4.15 \mathrm{MeV}$ (B.R. 21\%) and 4.20 MeV (B.R. 79\%); a weak doublet from ${ }^{234} \mathrm{U}$ may also be observed at $4.77 \mathrm{MeV}$ (B.R. 71.4\%) and $4.72 \mathrm{MeV}$ (B.R. 28.4\%). Two ${ }^{55} \mathrm{Fe}$ sources, emitting characteristic X-rays at $5.9 \mathrm{keV}$ and $6.4 \mathrm{keV}$, were also disposed in front of the germanium light detectors for calibration purposes.

\section{Bolometric performances}

The purpose of the bolometric test is to determine the $\mathrm{ZnSe}$ sample performances as a $0 v \mathrm{DBD}$ detector, that is the event discrimination capability and the energy resolution in the energy region of interest, around $2996 \mathrm{keV}$.
The detector was operated in a low-power dilution refrigerator $(20 \mu \mathrm{W}$ at $100 \mathrm{mK})$ at the cryogenics laboratory of Università degli Studi dell'Insubria, Como. In order to read the pulses produced by particles interactions, the thermistor must be supplied with a constant current flow: a steady bias voltage was applied to each thermistor, connected in series with two $200 \mathrm{M} \Omega$ load resistances at room temperature; the voltage signals were sent to lownoise amplifiers and then filtered with a Bessel $(125 \mathrm{~Hz}$ cut-off frequency). The data acquisition was performed by means of a dedicated software, also controlling the trigger: wave forms were sampled at $20 \mathrm{kHz}$ (1024 sample points in a $50 \mathrm{~ms}$ time window), digitized by a 12 bit LeCroy module and registered by a $500 \mathrm{MHz}$ Intel processor. The trigger is performed on-line by a dedicated software; data analysis is based on the optimal filter technique [6], which maximizes the signal to noise ratio (SNR).

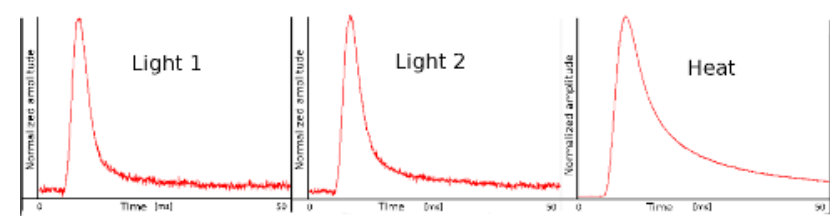

Figure 2. Example of a coincidence event: registered pulses on the heat channel and the two light detectors facing the scintillating crystal. Signal amplitudes are normalized.

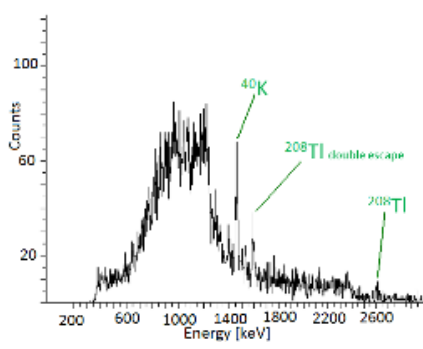

Figure 3. Heat channel: calibrated $\gamma$ spectrum. Sensitivity is $20 \mu \mathrm{V} / \mathrm{MeV}$ and FWHM energy resolution is $14 \mathrm{keV}$ at the $1460 \mathrm{keV}$ peak of ${ }^{40} \mathrm{~K}$, as expected from SNR.

Data were collected at $25 \mathrm{mK}$ base temperature. Typical signals time patterns are of the order of $50 \mathrm{~ms}$ for the $\mathrm{ZnSe}$ detector and $10 \mathrm{~ms}$ for the light detectors (figure 2). A background spectrum was registered, corresponding to $57 \mathrm{~h}$ live-time. Calibration of the heat channel was performed thanks to the natural radioactivity peaks of ${ }^{40} \mathrm{~K}$ and ${ }^{208} \mathrm{Tl}$, double-escape and photoelectric (figure 3); because of noise problems, we weren't able to discriminate the ${ }^{55} \mathrm{Fe}$ peaks on light detectors, however this is not essential for our main goals in this context. The energy resolution at $1460 \mathrm{keV}$ is about $14 \mathrm{keV} \mathrm{FWHM}$; this value is compatible with the theoretical energy resolution achievable with optimal filter, which is $0.27 \mu \mathrm{V}$ FWHM, corresponding to $13 \mathrm{keV}$ : so, at this level, no further contribution worsening the energy resolution is observable, such as trapping mechanisms in the crystal.

The two light detectors responses are comparable and an excellent event discrimination capability is achieved in the energy region of interest, where $\alpha$ s are clearly separated from $\beta, \gamma \mathrm{s}$ (figure 4); the $\mathrm{QF}$ is about 10, compatible 
with values found in previous tests with the same crystal [7].

Event discrimination is also possible using pulse shape analysis of the light channels. The Test Value Right (TVR) is defined as the average square difference between a pulse and the average "ideal" pulse, both optimally filtered; plotting this parameter as a function of pulse amplitude, three events families are discernible: pure ionization events on the light detector (TVR $<3), \alpha$ events $(3<$ TVR $<7.5)$ and $\beta, \gamma$ events (TVR $>7.5$ ) (figure 5).

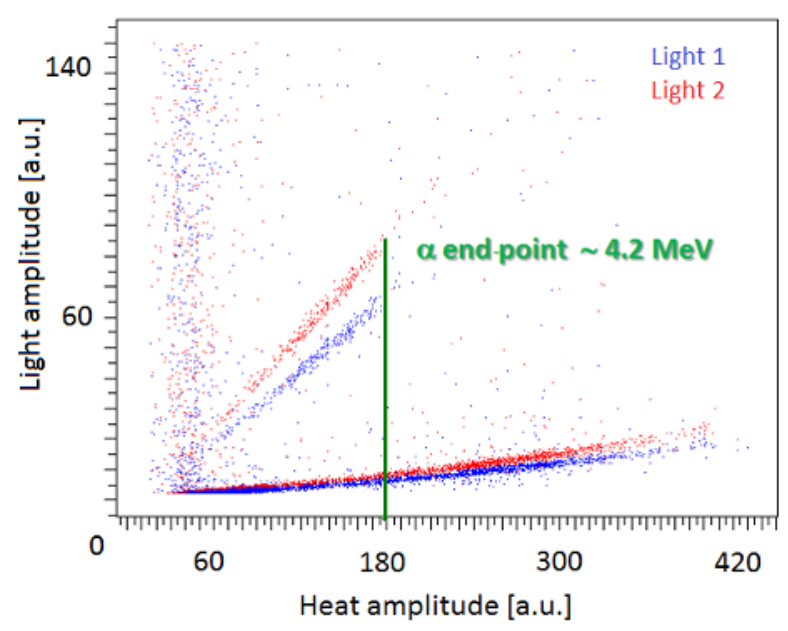

Figure 4. Plot of the coincidence events on the ZnSe heat channel and on light channel 1 (blue) and 2 (red). The light detectors responses are comparable and $\mathrm{QF}$ is around 10. The smeared $\alpha$ band, with end-point at $4.2 \mathrm{MeV}$, is clearly separated from $\beta, \gamma$ signals.
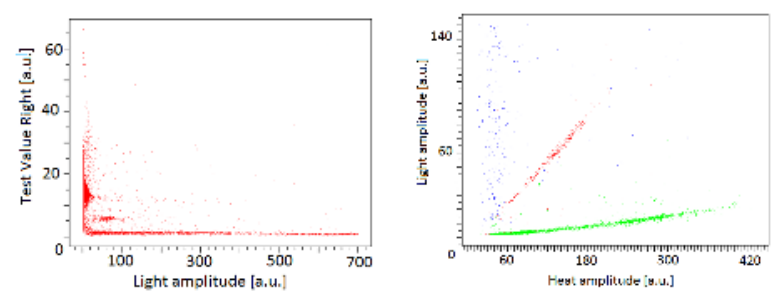

Figure 5. Plot of the light channel TVR as a function of pulse amplitude (left). The three events groups correspond to the different types of events in the heat/light scatter plot (right): pure ionization events on the light detector (blue, TVR $<3$ ), $\alpha$ s (red, $3<\mathrm{TVR}<7.5$ ) and $\beta, \gamma \mathrm{s}$ (green, TVR $>7.5$ ).

\section{Crystal characterization}

Optical and structure investigations were performed on a $10 \times 10 \times 1 \mathrm{~mm}^{3}$ slab, extracted from the same boule as the scintillating bolometer crystal: the main goal is to define parameters to select proper ZnSe energy absorbers.

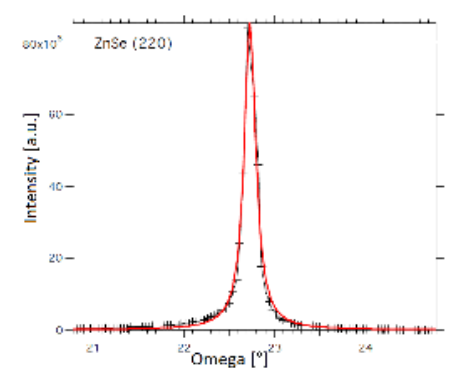

Figure 6. Transverse $\omega$ scan through the (220) Bragg peak. According to the Lorentzian fit, FWHM is $0.15^{\circ}$.

\subsection{Structural}

The structural properties of the sample were investigated using a 4-circle diffractometer mounted on a rotating copper anode $\left(\lambda\left(\mathrm{K}_{\alpha}\right)=1.54 \AA\right)$.

A Bragg peak was found along the direction normal to the sample surface at an angle $2 \theta=45.4^{\circ}$, corresponding to the (220) Bragg peak in the cubic phase. We could also find intense and narrow (111) and (311) Bragg peaks using the chi circle angular deviation (respectively at $81^{\circ}$ and $81.8^{\circ}$ ). We can thus deduce that the sample is a cubic single crystal with a lattice parameter $a=5.65 \AA$, and cut perpendicular to the $\langle 110\rangle$ direction. The transverse $\omega$ scan through the (220) Bragg peak (figure 6) has a FWHM value of $0.15^{\circ}$, indicating an angular mosaicity of about 4 minutes of angle, typical of a good crystallinity.

\subsection{Optical}

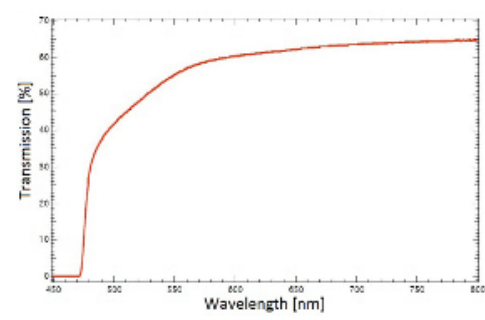

Figure 7. Transmitted intensity as a function of wavelength. The approximate value for room temperature gap is $2.63 \mathrm{eV}$.

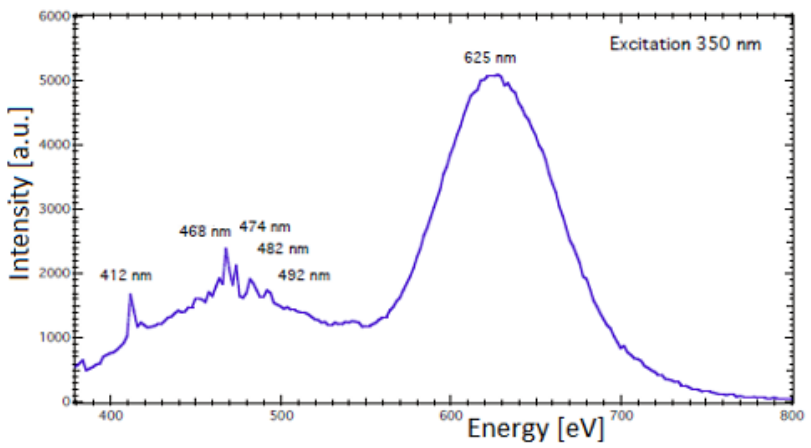

Figure 8. Luminescence spectrum at room temperature. The weak peaks in the low energy region are due to band-to-band and near-edge-band recombination; the broad intense peak at $625 \mathrm{~nm}$ is DAP contribution.

Transmission measurements were performed using a Cary 5000 UV-Vis Spectrophotometer equipped with a 


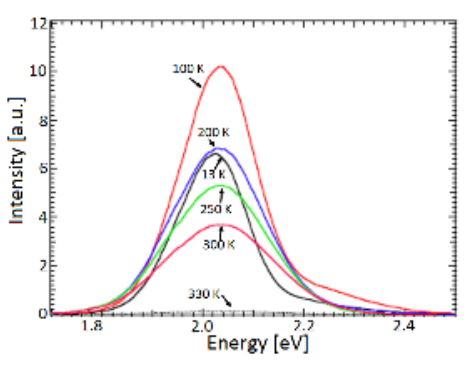

Figure 9. Intensity of the DAP peak at different temperatures. The loss of luminescence at high temperatures is due to the emptying of the donor levels and in agreement with the measured donor energy value.

Xe lamp. The transmitted intensity rises for wavelengths above $472 \mathrm{~nm}$, corresponding to an approximate value for the room temperature gap value of $2.63 \mathrm{eV}$ (figure 7).

The luminescence under UV excitation at $350 \mathrm{~nm}$ was measured at room temperature by means of a Triax 320 Horiba Jobin-Yvon spectrometer and a high power Xe lamp: the main peak is centered at $625 \mathrm{~nm}$ and corresponds to deep donor-acceptor pair (DAP) recombination, while the weaker peaks at lower energy are due to band-toband and near-edge-band recombination, correlated with Se vacancies [8] (figure 8). The DAP contribution was probed in a temperature range from $13 \mathrm{~K}$ and $350 \mathrm{~K}$, using a closed-cycle helium refrigerator (figure 9): the intensity is maximal around $100 \mathrm{~K}$ and shows a steep drop-off above $250 \mathrm{~K}$. This behaviour is related to the emptying of the donor levels through thermal excitation and is compatible with the measured donor energy level value of $21 \mathrm{meV}$, which was derived experimentally by studying the DAP peak energy shift as a function of laser power [9]. Indeed, the acceptor energy level of $647 \mathrm{meV}$ is in agreement with theoretical calculations involving $\mathrm{Zn}$ vacancies [10] and the DAP binding energy found from the model is about $41 \mathrm{meV}$. We thus have good indications that the observed DAP luminescence is related mainly to recombination between shallow donor and deep acceptor levels due to $\mathrm{Zn}$ Frenkel pairs.

\section{Conclusions}

We performed a complete characterization of a $\mathrm{ZnSe}$ scintillating crystal, in view of the realization of highly per- forming scintillating bolometers for Neutrinoless Double Beta Decay search, in the framework of the LUCIFER program. A prototype detector was operated aboveground at $25 \mathrm{mK}$ : good energy resolution (about $14 \mathrm{keV} \mathrm{FWHM} \mathrm{at}$ $1460 \mathrm{keV}$, compatible with SNR) and $\alpha$ versus $\beta, \gamma$ discrimination capability were achieved, thanks to double signal read-out and pulse shape analysis. The same ZnSe crystal underwent optical and X-ray diffraction measurements, showing the good crystallinity of the sample, which is a cubic single crystal, and that the main luminescence contribution is due to $\mathrm{Zn}$ point defects.

\section{Acknowledgements}

This work was partially supported by the LUCIFER experiment, funded by ERC under the FP7/2007-2013 grant agreement number 247115 . We thank Ioan Dafinei for providing us with the $\mathrm{ZnSe}$ samples.

\section{References}

[1] J.D. Vergados, H. Ejiri and F. Simkovic, Rep. Prog. Phys. 75, 106301 (2012)

[2] A. Giuliani, Physica B 280, 501 (2000)

[3] A. Giuliani et al., Proc. 5th Int. BEYOND 2010 Conference, Cape Town, South Africa, World Sci. (2010) p. 256

[4] C. Arnaboldi et al., 34, 344 (2011)

[5] J.W. Beeman et al., JINST 8, P05021 (2013)

[6] E. Gatti and P.F. Manfredi, Riv. Nuovo Cimento 9, 1 (1986)

[7] I. Dafinei et al., IEEE Trans. Nucl. Sci. 57, 1470 (2010)

[8] D. Etienne, J. Allègre, J. Chevrier, and G. Bougnot, Phys. Stat. Sol. 32, 279 (1975)

[9] E. Zacks and A. Halperin, Phys. Rev. B 6, 3072 (1972)

[10] F.C. Rong, W.A. Barry, J.F. Donegan and G.D. Watkins, Phys. Rev. B 54, 7779 (1996) 